increasing evidence of iron deficiency. Though the red cell volume was still within the normal range, it declined by $38 \%$ from the original measurement. This decline was probably due to the interaction of several factors. Most important was iron deficiency, doubtless aggravated by the frequent (usually daily) blood sampling as part of this patient's intensive respiratory investigation. A second factor could be the overall tendency for this patient's arterial oxygen tension to rise during his stay-amounting on average to an increase of $10 \mathrm{mmHg}$. The defect in folate metabolism is unlikely to be the explanation as the bone marrow was normal for most of this time.

Following correction of the iron deficiency the florid picture of folate deficiency reappeared.

In view of the rapidity and completeness of the response to folinic acid, the prompt reversion to normality on stopping TMP was not surprising. The stress of the recrudescence of his chest infection did not cause a relapse in the absence of TMP.

There is only one other published report suggesting disturbed folate metabolism in man during treatment with TMP. Kahn et al. (1968) have studied the effects of a high dose of TMP-sulphonamide combination (TMP $1000 \mathrm{mg}$ and sulphamethoxazole $4000 \mathrm{mg}$ daily). In a short - term study of ten subjects treated for 28 days, all cases developed changes in the morphology of the granulocytes of the peripheral blood with increased lobulation of nuclei. Five subjects developed bone marrow changes described as 'transitional megaloblastic erythroid change' and three others showed abnormalities of white cell precursors with giant metamyelocytes. One case had anaemia and one thrombocytopenia.

In their long-term studies of thirteen subjects given the same dose for 6-12 months, they found evidence of haematologic abnormality in six cases by examination of the peripheral blood but no bone marrow examinations were done. One of the six became anaemic and the other five had changes confined to the white cells or the platelet count. In one of these subjects they succeeded in reversing the peripheral blood change with folinic acid while TMP was continued, but they formed the impression that definitely 'supra normal' quantities were needed.

The currently recommended dose of TMP is only one third of that used in the above study by Kahn et al (1968). Our patient received the drug as currently recommended and is the first reported case of haematological abnormality with this dose.

\section{Acknowledgments}

We thank Dr A. Guz for his encouragement and advice, and Dr A. S. E. Fowle of the Wellcome Research Laboratories for providing a TMP - resistant mutant strain of Lactobacillus casei.

\section{References}

DACIE, J.V. \& Lewis, S.M. (1968) Practical Haematology, edn 4, Churchill, London.

HARWOOD, L. \& Forshaw, J. (1967) Vitamin B 12 $_{2}$ absorptior studies: Effect of parenteral non-radioactive Vitamin $B$ on serum levels of ${ }^{57} \mathrm{Co}$ Vitamin $\mathrm{B}_{12}$. Journal of Clinical Pathology, 20, 687.

Herbert, V. (1964) Studies in olate deficiency in man. Proceedings of the Royal Society of Medicine, 57, 377.

KAHN, S.B., FeIN, S.A. \& BrodSKy, I. (1968) Effects of trimethoprim on folate metabolism in man. Clinical Pharmacology and Therapeutics, 9, 550.

Raven, J.L., Walker, P.L. \& Barkham, P. (1966) A comparison of a radioisotope dilution-coated charcoal method and a micro-biological method (L. I eishmanii) for measuring $B_{12}$ in serum. Journal of Clinical Pathology, 19, 610.

TRINDER, P. (1956) The improved determination of iron in serum. Journal of Clinical Pathology, 9, 170.

Waters, A.H. \& Mollin, D.L. (1961) Studies on the folic acid activity of human serum. Journal of Clinical Patho$\log y, 14,335$.

\title{
Intracranial A-V malformation associated with cranial bruit and cervical venous hum
}

\author{
N. E. F. Cartlidge \\ M.B., M.R.C.P.
}

\author{
D. R. AYYAR \\ M.D., M.R.C.P.E.
}

\author{
M. LEE \\ M.B.,'M.R.C.P. \\ Department of Neurology, Royal Victoria Infirmary, Newcastle upon Tyne, 1
}

THE RECENT description (Hardison, 1968) of two patients with intracranial arteriovenous (A-V) malformations in whom loud cervical venous hums were audible, prompts us to describe the following case.

Reprint requests to: Dr N. E. F. Cartlidge. Ward 6, Royal Victoria Infirmary, Newcastle upon Tyne, 1

\section{Case report}

W.P., a 15-year-old boy, was admitted to Ward 6 of the Royal Victoria Infirmary on 11 April, 1969. At the age of 5 years he began to have 'bilious' attacks which subsided spontaneously after a few months. Paroxysmal attacks of headache began at 
the age of 11 years and these were preceded by flashing lights in front of both eyes and shimmering of vision. The latter would last for $15 \mathrm{~min}$ to be followed by a throbbing prostrating headache, first on one side and then the other. The headaches lasted for $24 \mathrm{hr}$, and they initially occurred once a month. Two years after their onset the character of the attacks changed. Instead of having shimmering of vision in front of the eyes, a right homonymous hemianopia would precede the headaches. This would last $15 \mathrm{~min}$ and would be followed by headache and vomiting. By this time the attacks were occurring once a fortnight and were seriously interfering with his schooling. His past history was unremarkable and there was no family history of migraine.

Physical examination revealed a blood pressure of $120 / 70 \mathrm{mmHg}$ in both arms and regular pulse of 80 per min. The optic fundi and the cranial nerves were normal. There was no abnormality in the motor, sensory or cerebellar systems. The reflexes were normal and equal on the two sides. The plantar reflexes were flexor.

On auscultation of the head a systolic bruit was heard over both temples and orbits being louder on the left side. In the sitting position, very loud venous hums were heard bilaterally in the supraclavicular areas. With the patient in the supine position, the venous hums, though diminished in intensity, were still very loud (Fig. 1).
Full blood count, ESR, serum electrolytes, blood urea, serum Wassermann reaction and Reiter CFT, EEG and ECG were all normal.

A diagnosis of an intracranial arteriovenous malformation was made. Plain X-rays of the skull showed a prominent venous channel on the left side running anteriorly from the occipital area. The markings due to the posterior branches of the middle meningeal vessels were also prominent on the left side (Fig. 2). Bilateral carotid angiograms were performed and these showed a large arteriovenous malformation involving the left cerebral hemisphere (Fig. 3).

$\mathrm{He}$ was not considered a suitable candidate for surgery.

\section{Discussion}

Intracranial A-V malformations commonly are first diagnosed after a subarachnoid or intracerebral haemorrhage (Henderson \& Gomez, 1967). However they may present with epilepsy, migraine or progressive hemiparesis. The classical sign of their presence is a cranial bruit, but this may be absent in up to a third of cases (Henderson \& Gomez, 1967), and even when present may vary in intensity and at times be difficult to hear. In addition, a cranial bruit may be heard in up to $60 \%$ of normal young children (Dalsgaard-Nielsen, 1939; Dodge, 1956; Allen \& Mustian, 1962), its incidence however dropping to $6 \%$ by the age of 15 (Wadia $\&$ Monkton, 1957).

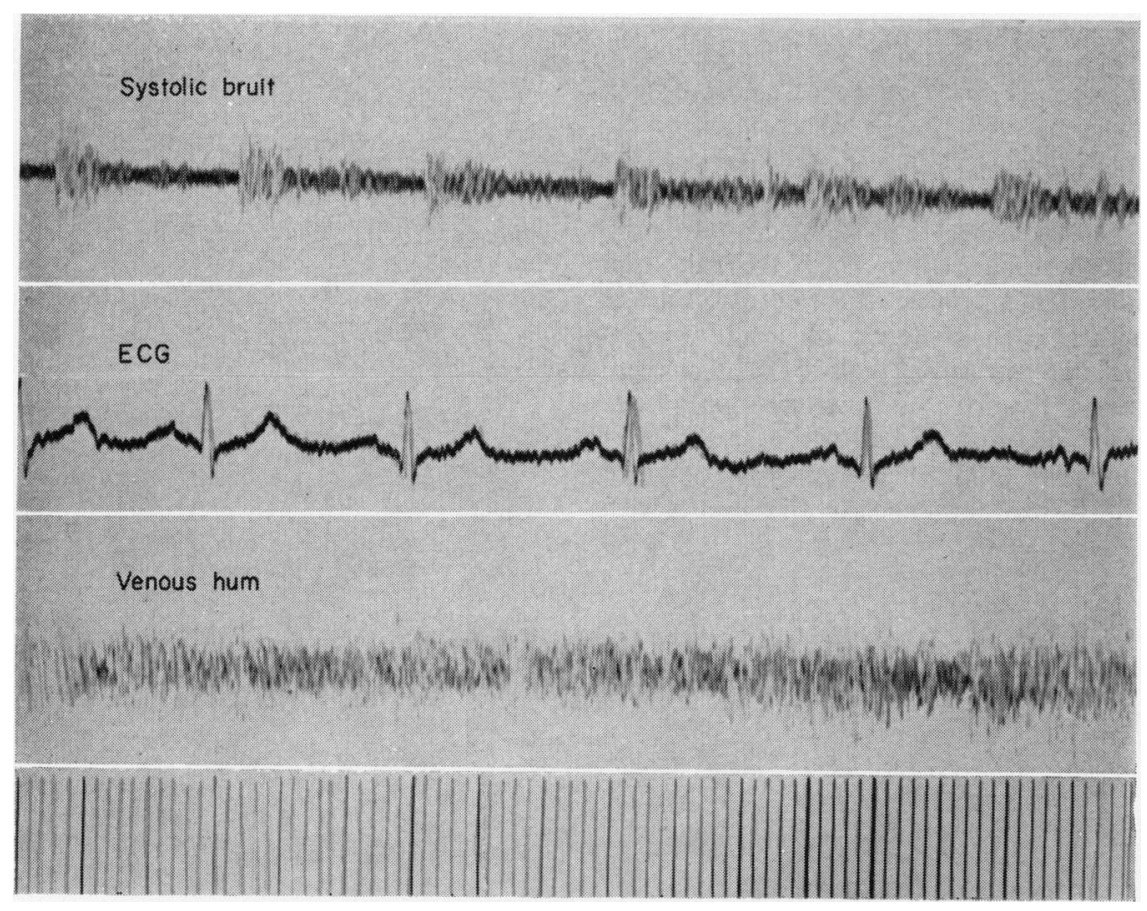

FIG. 1. Phonocardiogram showing the traces for systolic bruit, ECG and venous hum. 


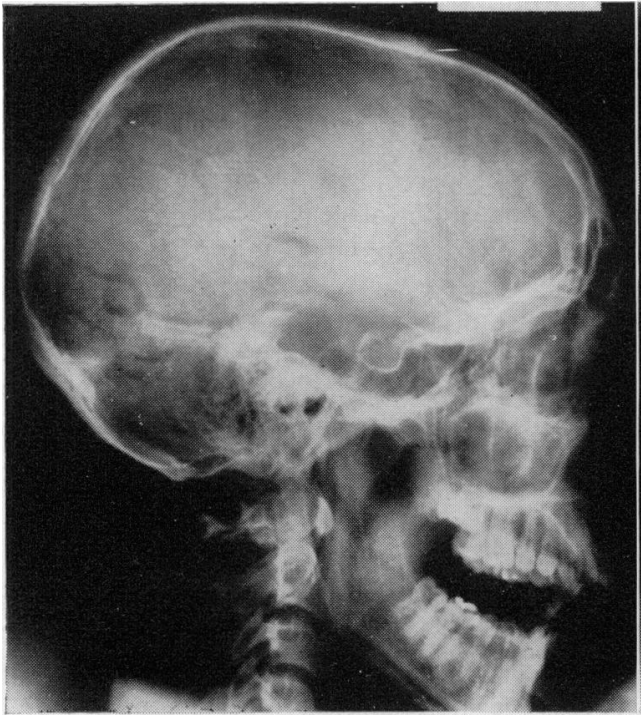

Fig. 2. Plain X-ray showing the markings due to the posterior branches of the middle meningeal vessels prominent on the left side.

The interest in this case lies in the presence of the cervical venous hum. In the sitting position this may be heard in the majority of children and young adults (Fowler \& Gause, 1964). On lying down however in the majority of cases it disappears.

Two previous cases of $\mathrm{A}-\mathrm{V}$ malformations in whom cervical venous hums were audible have been reported (Hardison, 1968) and in each of these cases, like our own, the hums were still audible with the patients supine.

The mechanism of cranial bruits in A-V malformations is recognized to be the increased cerebral blood flow and one must presume the venous hum is secondary to this. It is intriguing to speculate that patients who have the combination of a cranial bruit and a cervical venous hum will probably have relatively large malformations.

Certainly our case emphasizes the importance of auscultation of the skull and neck in all patients with migraine.

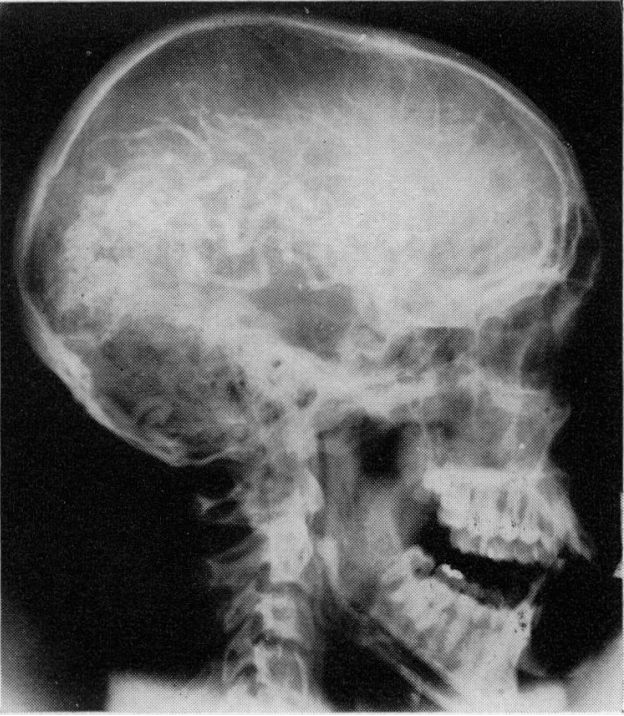

Fig. 3. Large arteriovenous malformation involving the left cerebral hemisphere.

\section{Acknowledgments}

It is a pleasure to thank Dr D. A. Shaw for all his help jn preparing this report.

\section{References}

Allen, N. \& Mustian, V. (1962) Origin and significance of vascular murmurs of the head and neck. Medicine (Baltimore), 41, 227.

DalsgaARD-Nielsen, T. (1939) Studies on intracranial vascular sounds. Acta Psychiatrica et Neurologica Scandinavica, 14, 69.

Dodge, H.W. (1956) Cephalic bruits in children. Journal of Neurosurgery, 13, 527.

Fowler, N. O. \& GAUSE, R. (1964) The cervical venous hum. American Heart Journal, 65, 135.

Hardison, J.E. (1968) Cervical venous hum: A clue to the diagnosis of intracranial arteriovenous malformation. New England Journal of Medicine, 278, 587.

Henderson, W.R., Gomez, R. DE R.L. (1967) Natural history of cerebral angiomas. British Medical Journal, 2, 571.

Wadia, N.H., Monkton, G. (1957) Intracranial bruits in health and disease. Brain, 80, 492. 